\title{
Maintenir l'information sous forme lexicale : une étude développementale ${ }^{1}$
}

\author{
Audrey Mazur-Palandre ${ }^{1,2, a}$ et Harriet Jisa ${ }^{3}$ \\ ${ }^{1}$ Laboratoire d'Excellence ASLAN (ANR-10-LABX-0081, de l'Université de Lyon dans le cadre du \\ programme « Investissements d'Avenir » (ANR-11-IDEX-0007) de l'État Français géré par l'Agence \\ Nationale de la Recherche) \\ ${ }^{2}$ Laboratoire ICAR (UMR 5191, CNRS, Université Lumière Lyon 2 et ENS de Lyon) \\ ${ }^{3}$ Laboratoire DDL (UMR 5596, CNRS et Université Lumière Lyon 2)
}

Résumé. L'objectif de cette étude est l'analyse des capacités linguistiques d'enfants et d'adolescents francophones natifs, en se focalisant sur le maintien de l'information lors de productions orales et écrites, dans une perspective développementale. Partant du constat que l'adolescence est une période clef du développement langagier, nous étudions les productions de trois groupes d'enfants (âge moyen : 10;9 (CM2), 12;7 (5ème) et 15;2 (3ème)). Parmi les compétences langagières à développer, l'organisation discursive apparait comme une importante étape acquisitionnelle de l'enfant de plus de 5 ans. La gestion des statuts informationnels des référents, l'introduction de l'information nouvelle ainsi que son maintien dans le texte font alors partis des capacités à acquérir pour produire un texte satisfaisant. Les analyses révèlent que les enfants, avec les années de scolarisation et l'habitude des exigences de l'écrit, maintiennent de plus en plus l'information ancienne sous forme lexicale, et ce davantage en production écrite qu'en production orale. Ce maintien lexical se réalise également de manière de plus en plus élaborée : si les jeunes enfants se contentent d'utiliser la répétition, les enfants plus âgés usent de la métonymie ou de l'hyperonymie. Ces résultats seront discutés dans une perspective développementale, en prenant en considération le contexte de production.

\begin{abstract}
Maintaining information with a lexical form: a developmental study. The aim of this study is to analyze the linguistic abilities of French children and adolescents by focusing on maintaining information during spoken and written text production, in a develomental perspective. Much development in the ability to produce a monologue text occurs during childhood and adolescence. For this reason, we have studied the productions of three groups of children (mean age: 10;9 (CM2), 12;7 (5ème) and 15;2 (3ème)). Among the linguistic abilities that develop during this period, discursive organisation constitutes an important
\end{abstract}

\footnotetext{
${ }^{a}$ Auteur de correspondance : audrey.mazur_palandre@ens-lyon.fr
} 
acquisitional step of children after 5. Manipulating the informational status of referents, introducing new information and maintaining it in the text are competencies necessary for the production of a well-formed production. Acquisition of school language and increasing school experiences have an impact on children's linguistic choices. Analyses revealed indeed that children increasingly maintained information with a lexical form and this is observed more in written production than in spoken production. Moreover, the use of a lexical form to maintain old information is becomes more elaborated: if young children essentially used repetition, older children used metonymy or hyperonymy. These results will be discussed in a developmental perspective, by taking into account the context of production.

\section{Introduction}

L'acquisition et le développement du langage est au centre des préoccupations sociétales, dans la mesure où la maitrise de la langue est garante de réussite scolaire, professionnelle et sociale. C'est dans cette mesure que l'Éducation Nationale, dans son programme scolaire à l'école maternelle, donne une place primordiale au langage qui est "réaffirmé comme condition essentielle de la réussite de toutes et de tous » (B.O. officiel spécial $n^{\circ} 2$ du 26 mars 2015). La structuration du langage constitue alors une priorité de l'école dont l'un des objectifs est ainsi de permettre « à chacun d'aller progressivement au-delà de la simple prise de parole spontanée et non maîtrisée pour s'inscrire dans des conversations de plus en plus organisées » (B.O. officiel spécial $\mathrm{n}^{\circ} 2$ du 26 mars 2015). L'enrichissement de la production langagière passe alors, par exemple, par la production plus importante de formes lexicales que les enfants adaptent aux contextes de production (entre autres, Katzenberger, 2004 ; Mazur-Palandre, 2012) ou encore par la production de propositions ${ }^{2}$ plus complexes faisant appel à des structures plus rares (entre autres, Berman, 2008 ; Berman et Verhoeven, 2002 ; Jisa, 2004 ; Mazur-Palandre, 2010 ; Mazur-Palandre et Jisa, 2012 ; Ravid, 2005). Etre linguistiquement mature (proficient speaker) suppose la possession d'un répertoire linguistique, qui englobe un large éventail de registres et de genres, ainsi que la capacité de s'adapter aux contextes communicationnels (Berman, 2008 ; Berman et Ravid, 2009 ; Tolchinsky, 2006).

Cet article se concentre ainsi sur le développement langagier de l'individu, et plus précisément sur le passage du statut de native speaker à celui de proficient speaker en production (Berman, 2008), c'est-à-dire au statut du locuteur performant ayant les capacités de gérer des situations langagières diverses et complexes l'obligeant à manipuler ses connaissances linguistiques, cognitives, discursives et culturelles, avec lesquelles il doit jongler efficacement (Berman, 2008). Parmi les compétences langagières à développer pour parvenir au statut de proficient speaker, l'organisation discursive apparait comme une des plus importantes étapes acquisitionnelles de l'enfant de plus de 5 ans (Hickmann, 2002). La gestion des statuts informationnels des référents, l'introduction de l'information nouvelle ainsi que son maintien dans le texte font partis des capacités pour produire un texte satisfaisant.

Ces aspects ont été sujets de plusieurs études antérieures (De Weck, 1991 ; De Weck et Schneuwly, 1994 ; Hickmann, 2002, 2003 ; Kail et Hickmann, 1992 ; Schneuwly, 1988 ; Schneuwly, Rosat et Dolz, 1989). Néanmoins, peu de travaux se focalisent sur le maintien de l'information dans une perspective développementale prenant en considération la différenciation des modalités orale et écrite, comme le fait cette présente étude. Cet article est d'autant plus novateur qu'il se focalise sur un type de maintien spécifique : le maintien sous forme lexicale, qui, s'il est peu étudié, semble être pourtant un indicateur de texte adéquat aux attentes scolaires. Ainsi, à partir de quel âge les enfants utilisent la forme lexicale, appelée de manière naturelle pour la fonction d'introduction d'une information 
nouvelle, pour maintenir une information ? Est-ce qu'un contexte de production favorise le maintien de l'information sous forme lexicale ? À partir de quel âge et dans quel contexte les enfants utilisent une nouvelle forme le syntagme nominal lexical (SNL), pour une ancienne fonction, maintenir une information?

\section{Contextes de production}

\subsection{La modalité de production}

Pour cette étude, le contexte de production renvoie aux deux modalités du langage, orale et écrite, qui possèdent des caractéristiques propres (Blanche-Benveniste, 1983, 1990, 2000 ; Fayol, 1997 ; Gadet, 1996 ; Gayraud, 2000). À l'oral, les individus vouent moins de temps à la planification; ils doivent mobiliser rapidement les informations relatives à un message (Chafe, 1994). Prototypiquement, la modalité écrite assouplit cette contrainte temporelle grâce à un rythme de production plus lent et plus modulable. Les locuteurs bénéficient, à l'écrit, d'un temps de planification potentiellement supérieur ce qui facilite, par exemple, la recherche lexicale (Fayol, 1997), la production d'un lexique plus riche ou la production de formes syntaxiques exigeant un traitement cognitif plus lourd (Gayraud, Jisa et Viguié, 2001; Jisa, 2004).

Par ailleurs, l'oral impose aux locuteurs une pression communicative que la modalité écrite n'exige pas. En effet, le locuteur, pour mener à bien son intervention, doit - en plus de mobiliser les informations pertinentes au message, les planifier et les encoder, capter l'attention des destinataires - prévenir et empêcher les interruptions des autres participants. D'un autre côté, la modalité écrite implique un registre académique ainsi qu'une norme rédactionnelle exigeante. Ceci implique alors la mobilisation de formes lexicales et de structures syntaxiques rares, appartenant à un registre élevé. Elle impose également une pression informative importante ; les scripteurs se doivent de fournir un maximum d'informations, ce qui peut se concrétiser par la production massive de lexique. Nous disons alors que l'écrit apparaît comme un état plus planifié de la langue que l'oral.

Enfin, la communication écrite ne propose pas un cadre aussi collaboratif que la communication orale. Le « savoir partagé » (« common ground») est alors plus difficile à établir. Cependant, à l'écrit, les individus pourraient exploiter le rythme de production lent de la production écrite pour se mettre à la place du destinataire ; ce qui serait rendu plus difficile à l'oral, caractérisé comme ayant un rythme de production plus rapide.

Les modalités orale et écrite divergent donc sur de nombreux points et ces différences ont des conséquences sur les choix linguistiques des individus. Ainsi, par exemple, les unités phrastiques, à l'oral, sont de petite taille étant donné le rapide temps de traitement et le caractère éphémère de l'oral. Ces unités ne véhiculent qu'une seule information à la fois en sachant que l'information nouvelle est, en général, placée préférentiellement en position post verbale et que la position sujet est remplie par les pronoms. La modalité orale met enjeu la contrainte du sujet léger. Elle se manifeste par l'évitement de syntagme nominal lexical en position sujet, fonction syntaxique réservée à l'information ancienne et donc à des formes exprimant l'accessibilité telles que le pronom (Chafe, 1994). Néanmoins, l'écrit violerait cette contrainte du "sujet léger» ("the light subject constraint», notion de Chafe, 1994) en facilitant la production de sujets lexicaux pouvant introduire un nouveau référent.

\subsection{Le type de texte}

En plus des deux modalités du langage, deux types de textes sont distingués : la narration et le discours logico-scientifique (expositif), reflétant des modes de pensée bien distincts 
(Bruner, 1986 ; Ravid et Berman, 2010 ; Mosenthal, 1985), ce qui a des répercussions sur les choix linguistiques des locuteurs/scripteurs (Bruner, 1986 ; Ravid et Berman, 2010).

Le type de texte narratif (narration personnelle) se définit comme une suite de faits détaillés dans l'ordre chronologique (Berman et Slobin, 1994 ; Hickmann, 2003). Cette organisation temporelle et aussi causale (Aksu-Koç et Küntay, 2001 ; Jisa et Mazur, 2006) facilite la mise en texte d'un évènement (Fayol, 1997). En revanche, le texte expositif est une production dans laquelle les locuteurs créent une structure thématique, exposent des faits ou des idées théoriques et des interprétations de ces derniers (Boscolo, 1990 ; Britton, 1994 ; Katzenberger, 2004 ; Tolchinsky, Perera, Argerich et Aparici, 1999 ; Ravid et Berman, 2010). Ce type de texte, qui dérive de l'écrit académique (Mosenthal, 1985), requiert un déploiement de relations logiques, et non pas chronologiques, entre les arguments (Britton, 1994 ; Mosenthal, 1985 ; Ravid, 2005).

De plus, dans le texte narratif, les locuteurs/scripteurs sollicitent des souvenirs personnels qui impliquent la mobilisation de référents spécifiques qu'ils maintiennent tout le long de leur production. Par opposition, le type de texte expositif fait appel à des informations d'ordre générique (Berman et Nir-Sagiv, 2007 ; Katzenberger, 2005 ; Mosenthal, 1985), qu'ils introduisent, puis qu'ils développent et discutent. Cela est lié à la pression informative de ce type de texte et à son but communicationnel, développer des thématiques.

Enfin, le type de texte expositif, beaucoup plus que le type narratif, exige la mobilisation du registre académique (type de registre utilisé, par exemple, dans les écrits scolaires et universitaires, ce qui se traduit par la mobilisation de diverses relations rhétoriques de haut niveau telles que la gestion de l'agencement des idées (Bosocolo, 1990 ; Mosenthal, 1985). Ce type de texte dispose alors de formes linguistiques complexes et plus coûteuses cognitivement comme des «sujets lourds", à savoir des sujets sous formes lexicales (Ravid, 2005). Il requiert la mise en relation de deux niveaux : le niveau du contenu et celui de la rhétorique (Britton, 1994 ; Katzenberger, 2004 ; Mosenthal, 1985). De ce fait, sa planification et son organisation sont beaucoup plus exigeantes que celles du texte narratif (Boscolo, 1990).

Par définition, ces deux types de textes impliquent des caractéristiques très différentes ayant des répercutions sur les choix linguistiques des locuteurs/scripteurs (Bruner, 1986 ; Ravid et Berman, 2010). Le texte narratif se caractérise, entre autres, par l'utilisation de formes spécifiques (pronoms personnels), des noms concrets, des propositions simples et courtes (entre autres, Berman et Nir-Sagiv, 2007 ; Ravid, 2005). En revanche, le texte expositif fait appel à des formes génériques (pronoms impersonnels et génériques), des noms abstraits, des propositions plus longues et des structures syntaxiques dites plus complexes (passif, subordination non finie, par exemple) (entre autres, Berman et NirSagiv, 2007 ; Ravid, 2005).

\section{Statut informationnel}

\subsection{Le continuum information Nouvelle / Ancienne}

Produire un message implique l'énonciation de diverses informations qui peuvent être nouvelles ou anciennes (Khorounjaia et Tolchinsky, 2004). Tout acte communicationnel exige des locuteurs la capacité de manipuler ces deux types d'information. L'information nouvelle se caractérise comme un référent qui n'a pas été mentionné précédemment dans le discours et qui n'est pas accessible par l'interlocuteur dans la situation d'énonciation (Allen, Skarabela et Hughes, 2008; Chafe, 1994; Halliday et Hasan, 1976/1989). L'information ancienne correspond à une information retrouvable par l'interlocuteur dans le discours ou dans le contexte (Allen et al., 2008 ; Halliday et Hasan, 1976; Chafe, 1994). 
Pour introduire et maintenir une information, les locuteurs/scripteurs ont recours à des outils linguistiques et cohésifs (entre autres, Allen et al., 2008 ; Ariel, 1996 ; Fox, 1987 ; Khorounjaia et Tolchinsky, 2004 ; Levelt, 1989 ; Sanford et Garrod, 1981) : un locuteur coopératif marque syntaxiquement l'accessibilité d'un référent (Levelt, 1989). L'encodage d'un référent sous forme de SNL, qui est de faible accessibilité ("low accessibility » Khorounjaia et Tolchinsky, 2004:88, notion d'Ariel, 1996), est utilisé pour introduire une information nouvelle (Allen et al., 2008 ; Fox, 1987). En revanche, les pronoms, qui sont de haute accessibilité («high accessibility», Khorounjaia et Tolchinsky, 2004:88), sont utilisés pour encoder des référents actifs donc anciens (Allen et al., 2008 ; Fox, 1987). Le fait que le pronom encode préférentiellement l'information ancienne et le SNL une information nouvelle semble être un principe universel : il s'agit du traitement naturel de l'information (Du Bois, 1987, 2003).

Des principes universaux régentent les langues. Le premier principe universel est celui selon lequel l'information ancienne se situerait préférentiellement au début d'une proposition alors que l'information nouvelle se situerait plutôt en fin de proposition (Du Bois, 1987 ; Jisa, 2000 ; Khorounjaia et Tolchinsky, 2004 ; Lambrecht, 1994). Le choix d'introduire l'information nouvelle en position non sujet est gouverné par des contraintes discursives pesant sur les choix linguistiques (Du Bois, 1987, 2003 ; Khorounjaia et Tolchinsky, 2004). À ce premier principe, une seconde tendance s'ajoute : la contrainte du "sujet léger" ("the light subject constraint», notion de Chafe, 1994). Les locuteurs/scripteurs en production combleraient la position syntaxique sujet avec de l'information ancienne qui serait de plus encodée, le plus souvent, sous la forme d'un pronom. La décision d'encoder un référent sous une forme particulière dans une position syntaxique spécifique relève de facteurs syntaxiques, pragmatiques, discursifs mais également cognitifs. En termes de cognition, l'information nouvelle exige plus de ressources cognitives que l'information ancienne dans la mesure où cette dernière est active et donc plus ou moins immédiatement accessible (Chafe, 1994; Khorounjaia et Tolchinsky, 2004), qui est de surcroit encodée par une forme lexicale. C'est ainsi, étant donné le coût cognitif que mobilise l'introduction d'une information nouvelle, qu'une contrainte conversationnelle existe : la contrainte d'une seule nouvelle idée par proposition («the one new idea constraint», Chafe, 1994:119). Les locuteurs/scripteurs ne transmettraient au maximum qu'une information nouvelle par proposition (Du Bois, 1987, 2003 ; Chafe, 1994 ; Khorounjaia et Tolchinsky, 2004).

\subsection{Le maintien de l'information sous forme lexicale}

L'information ancienne s'encode ainsi préférentiellement par le biais d'une forme pronominale en position sujet (1).

(1) Les affaires de ma copine avaient disparues et personne ne savait où elles étaient passées. (3ème $n^{\circ} 05$, narratif écrit, Ordre écrit/oral)

Néanmoins, la langue offre une autre possibilité : le maintien de l'information par le biais d'une forme lexicale. Halliday et Hasan (1976/1989) définissent ce type de maintien référentiel en terme de cohésion lexicale, et plus spécifiquement de réitération. Parmi les possibilités de réitération apparaissent (a) la répétition (2), (b) la synonymie (3) et (c) l'hyperonymie (4).

(2) Il y avait un gros champignon poussant près d'elle, arrivant à peu près à se hauteur [...]. Elle se mit sur la pointe des pieds et jeta un coup d'œil par-dessus le champignon. (Halliday et Hasan, 1976/1989)

(3) J'ai lu le livre de Marc Levy. Le bouquin est très sympa!

(4) J'ai pu caresser des tigres. Ces félins sont très impressionnants. 


\subsection{Le statut informationnel dans une perspective développementale}

Dans l'exemple (5) qui suit, l'enfant introduit l'information nouvelle par le biais d'une forme lexicale : la violence, puis la triche et enfin le vol.

(5) La violence n'est pas bien du tout. La triche en classe ne sert à rien. Le vol est très mal. (CM2 $n^{\circ} 24$, expositif écrit, E/O)

Ce participant introduit trois informations nouvelles, ce message en est-il pour autant satisfaisant? L'individu renouvelle certes l'apport d'informations nouvelles dans son texte mais il ne maintient pas ces référents. Un message pour être satisfaisant doit comporter deux types d'informations: de nouveaux et d'anciens référents (Charolles, 1978 ; givennew contract, Clark et Haviland, 1977 ; Khorounjaia et Tolchinsky, 2004 ; Reinhart, 1980) impliquant alors effectuer un travail de conservation/progression (Schneuwly, 1988:41). Produire un texte cohérent exige une symbiose et un équilibre entre l'introduction d'informations nouvelles et la continuité référentielle qui reposerait sur quatre règles principales :

(1) la méta-règle de répétition (Bellert, 1970 ; Charolles, 1978 ; Reinhart, 1980) selon laquelle le texte doit comporter «dans son développement linéaire des éléments à récurrence stricte » (Charolles, 1978:14);

(2) la méta-règle de progression (Charolles, 1978:20) qui implique que pour assurer la cohérence d'un texte "il faut que son développement s'accompagne d'un apport sémantique constamment renouvelé »;

(3) la méta-règle de non-contradiction (Charolles, 1978 ; Reinhart, 1980) qui implique que le texte produit soit cohérent, "il faut que son développement n'introduise aucun élément sémantique contredisant un contenu posé ou présupposé par une occurrence antérieure ou déductible de celle-ci par inférence. " (Charolles, 1978:22);

(4) la méta-règle de relation (Charolles, 1978 ; Reinhart, 1980) qui introduit l'idée que pour avoir un texte cohérent, " il faut que les faits qu'ils dénotent dans le monde représenté soient reliés » (Charolles, 1978:31).

Ainsi, produire un message cohérent ne résulte pas seulement de l'introduction de nouveaux éléments. Un locuteur expert introduit une information nouvelle, la développe en la maintenant, fait des prédications puis passe à une information nouvelle, qu'il développe, etc.. Le fait de maintenir dans le but de développer son texte est une aptitude à acquérir. Dans une étude antérieure, Mazur-Palandre et Jisa (2012) montrent que les enfants les plus avancés dans le cursus scolaire introduisent certes des informations nouvelles mais les développent et les travaillent alors que les enfants plus jeunes se contentent de lister de nouveaux référents. Dans tout texte, le locuteur doit donc jongler entre apport d'informations nouvelles et maintien d'informations connues à partir desquelles des prédications sont faites.

Cette organisation discursive " constitue l'une des facettes les plus importantes du langage chez l'enfant après six ans "(Hickmann, 2002:181). Durant cette période, il apprend à maîtriser un système complexe qui doit mettre en relation les formes et les fonctions des unités linguistiques tout en prenant en considération le contexte et en évaluant la connaissance mutuelle. Les enfants doivent alors intégrer deux types de connaissances : celles relatives aux règles du contenu d'un message (soit la cohérence) et celles relatives aux règles gouvernant le flux de l'information entre les propositions d'une production (soit la cohésion). Le locuteur/scripteur, pour réguler l'information dans un discours, doit gérer trois aspects : (a) le statut informationnel des éléments ; (b) les connaissances des destinataires ; (c) la différenciation des informations essentielles et secondaires. Un des outils cohésifs permettant la gestion de ces trois points est la référence. L'utilisation et la gestion du système référentiel est une acquisition tardive (Hickmann, 2003). Les enfants sont capables, en compréhension, de distinguer la continuité et la discontinuité référentielle d'une production assez tôt (Hickmann et Hendriks, 1999). 
Cependant, la production des outils relatifs à la référence relève d'une interaction complexe entre les fonctions syntaxiques, sémantiques et pragmatiques des formes. Les analyses révèlent que, si les référents sont très clairement établis, alors la cohésion est assez bien gérée dès l'âge de 9/10 ans (Pellegrini, Galda et Rubin, 1984). Néanmoins, en cas de connaissance partagée limitée, la gestion des marques concernant le statut informationnel est entravée (Kail et Hickmann, 1992). L'acquisition de la référence, à l'écrit comme à l'oral, se prolongerait au moins jusqu'à 10/11 ans (Hickmann, 2003 ; Hickmann et al., 1996 ; Hickmann et Hendricks, 1999).

\section{Hypothèses}

Cet article se concentre sur le maintien de l'information ancienne, et plus spécifiquement sur le maintien d'un référent sous forme lexicale, qui apparaît comme un choix non naturel. La forme linguistique choisie pour encoder un référent dépend de son statut informationnel dans le discours (Ariel, 1996 ; Khorounjaia et Tolchinsky, 2004 ; Levelt, 1989 ; Sanford et Garrod, 1981). Un nouveau référent, qui est de faible accessibilité, est encodé sous la forme de SNL, alors qu'un élément actif, donc ancien, est préférentiellement encodé sous forme pronominale. Les participants de notre étude devraient suivre cette tendance et la proportion de l'information ancienne sous forme pronominale serait donc plus importante que celle de l'information ancienne sous forme lexicale (Hypothèse 1).

Nous supposons un effet du NIVEAU SCOLAIRE sur la proportion des SN maintenant une information sous forme lexicale. Avec l'expérience, les individus de notre étude maintiennent certainement de plus en plus une information sous forme lexicale. Les locuteurs/scripteurs apprennent à utiliser le SNL, comme outil rhétorique, pour maintenir une information. La proportion de l'information ancienne sous forme lexicale aurait donc une proportion plus importante dans les textes des enfants plus âgés (Hypothèse 2).

Si notre hypothèse - selon laquelle la proportion du maintien sous forme lexicale augmente avec les années de scolarisation - se confirme, alors le maintien sous forme lexicale apparaît comme un signe de maturité. Les enfants de notre étude doivent suivre une trajectoire, qui aboutira au comportement linguistique de scripteurs experts (De Weck, 1991 selon Moirand, 1975), à savoir maintenir une information sous une forme lexicale différente de la première apparition. Est ainsi observée la proportion des SNL maintenant une information impliquant des changements linguistiques par rapport à la mention précédente (Mon chat s'est enfui, ce petit félin me manque), versus les SNL maintenant une information en n'impliquant pas de changement linguistique par rapport à la mention précédente (Mon chat s'est enfui, mon chat me manque). Les enfants avec l'expérience vont ainsi sans doute de plus en plus modifier la forme des SNL qu'ils maintiennent (Hypothèse 2bis).

Nous attendons également un effet du contexte de production (MODALITÉ et TYPE DE TEXTE). Les participants de notre étude privilégieraient le maintien sous forme lexicale pour les textes expositifs et écrits. En effet, nous pouvons penser que la substitution lexicale se réalise davantage en production expositive qu'en production narrative, ce qui serait dû au registre académique de ce texte ainsi qu'à sa pression informationnelle. De plus, l'écrit, par son rythme de production lent, facilite l'accès lexical et donc la mobilisation et la production de SNL. Cette modalité de production est sans doute celle permettant le plus aux individus d'aller à l'encontre des principes naturels de la transmission de l'information, à savoir maintenir une forme de préférence par un pronom. La proportion de l'information ancienne sous forme lexicale serait plus importante dans les textes écrits et expositifs (Hypothèse 3).

Nous nous attendons également à ce que les individus maintiennent plus fréquemment une information en variant la forme linguistique à l'écrit et en texte expositif, qu'à l'oral et qu'en texte narratif. La proportion de l'information ancienne sous forme lexicale 
impliquant un changement linguistique par rapport à la première mention serait ainsi plus importante dans les textes écrits et expositifs (Hypothèse 3bis).

Enfin, nous suggérons que les individus ayant produit dans l'ordre écrit/oral maintiennent plus l'information sous forme lexicale que les individus ayant produit dans l'ordre inverse; et ce pour les raisons évoquées précédemment: les formes lexicales mobilisées à l'écrit restent activées lors de la production orale et les locuteurs les utilisent de nouveau. La proportion de l'information ancienne sous forme lexicale serait ainsi plus importante dans les textes des enfants ayant produit dans l'ordre écrit/oral (Hypothèse 4).

Nous pouvons également penser que les individus appartenant à l'ordre de passation écrit/oral maintiennent plus une information sous forme lexicale en variant la forme. La proportion de l'information ancienne sous forme lexicale impliquant un changement linguistique par rapport à la première mention serait plus importante dans les textes des enfants ayant produits dans l'ordre écrit/oral (Hypothèse 4bis).

\section{$5 \quad$ Méthodologie}

\subsection{Participants}

La collecte des données a été réalisée dans le cadre d'un projet ANR (responsable scientifique : Harriet Jisa) et comprenait 3 groupes d'âges (10-11 ans ; 12-13 ans et 14-15 ans, Tableau 1) scolarisés dans trois écoles primaires et un collège ZEP/REP d'une commune de l'est-lyonnais.

Tableau 1. Description des groupes d'individus

\begin{tabular}{|llll|}
\hline & CM2 & $5^{\text {ème }}$ & $3^{\text {ėme }}$ \\
\hline Âge & & & \\
Moyenne & $10 ; 9$ & $12 ; 7$ & $15 ; 2$ \\
Intervalle & {$[10 ; 00-11 ; 10]$} & {$[12 ; 03-14 ; 04]$} & {$[14 ; 06-16 ; 07]$} \\
\hline Nombre total d'individus & 44 & 43 & 45 \\
Nombre total de filles & 22 & 22 & 22 \\
Nombre total de garçons & 22 & 21 & 23 \\
\hline
\end{tabular}

La sélection des individus a été menée par le biais d'un questionnaire qui avait 3 objectifs principaux : (1) connaître la Catégorie Socio-Professionnelle (CSP, définie selon les grilles de l'INSEE) des parents des élèves pour établir une homogénéité de milieu ; (2) connaître le pays de naissance des enfants et des parents ainsi que leur environnement linguistique afin de sélectionner des monolingues francophones natifs; (3) écarter les individus ayant un développement langagier atypique.

\subsection{Collecte des données}

Les données des individus ont été collectées selon des procédures strictes avec des moyens techniques permettant d'obtenir des données chronométriques (tablettes graphiques, enregistrements numériques). L'objectif était d'obtenir de la part de chaque individu la production de deux types de textes (expositifs et narratifs) à l'oral et à l'écrit. Une thématique a été imposée, celle du conflit, dans la mesure où ce thème fait davantage produire que tout autre thème (Gayraud, 2000 ; Viguié-Simon, 2001). Afin de faire produire les individus sur cette thématique, une vidéo muette (créée par une équipe de la Télévision Éducative d'Israël pour une étude antérieure, le Projet Spencer, porteur Ruth Berman) leur a été présentée. Cette vidéo est «moralement inoffensive et visible » (Viguié- 
Simon, 2001:46) et présente une série de clips sur «les problèmes entre les gens » en milieu scolaire (tricherie, disputes, etc.).

Quatre textes étaient attendus de chaque individu : narratif oral, narratif écrit, expositif oral, expositif écrit. Pour la production narrative, il a été demandé aux enfants de raconter une histoire/une dispute qu'ils avaient pu avoir. Pour les textes expositifs, il a été demandé aux enfants d'exposer leur connaissance quant aux « problèmes entre les gens ». L'ordre de la production des textes a été contrôlé (4 groupes, Tableau 2) et contre balancé dans la mesure où des études pilotes ont révélé un effet significatif de l'ordre de production pour certains critères d'analyse (Jisa, 2004 ; Jisa et Mazur, 2006 ; Gayraud et al., 2001 ; MazurPalandre, 2015 ; Mazur-Palandre et Jisa, 2013).

Tableau 2. Ordre de production passage

\begin{tabular}{|c|c|c|c|}
\hline \multicolumn{2}{|c|}{ Session 1} & & Session 2 \\
\hline \multirow{4}{*}{ 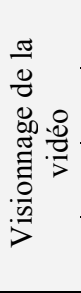 } & A & $\begin{array}{l}\text { Narratif oral - Questionnaire - } \\
\text { Narratif écrit }\end{array}$ & $\begin{array}{l}\text { Expositif oral - Questionnaire - } \\
\text { Expositif écrit }\end{array}$ \\
\hline & B & $\begin{array}{l}\text { Narratif écrit - Questionnaire - } \\
\text { Narratif oral }\end{array}$ & $\begin{array}{l}\text { Expositif écrit - Questionnaire - } \\
\text { Expositif oral }\end{array}$ \\
\hline & $\mathrm{C}$ & $\begin{array}{l}\text { Expositif oral - Questionnaire - } \\
\text { Expositif écrit }\end{array}$ & $\begin{array}{l}\text { Narratif oral - Questionnaire - } \\
\text { Narratif écrit }\end{array}$ \\
\hline & D & $\begin{array}{l}\text { Expositif écrit - Questionnaire - } \\
\text { Expositif oral }\end{array}$ & $\begin{array}{l}\text { Narratif écrit - Questionnaire - } \\
\text { Narratif oral }\end{array}$ \\
\hline
\end{tabular}

La collecte s'est organisée sur deux sessions. Une semaine séparait les deux sessions et, entre les deux productions de textes d'une même session, des tâches de distraction (sous forme de questionnaires) étaient proposées.

\subsection{Codage des données}

Pour analyser et coder les productions, le logiciel CLAN a été utilisé selon les conventions CHILDES (Child Language Data Exchange System, http://childes.psy.cmu.edu/). L'information ancienne a été codée, par opposition à l'information nouvelle. Comme dit précédemment, un syntagme a été considéré comme portant une information nouvelle quand le référent n'a pas été mentionné précédemment dans le discours et qu'il n'est pas accessible par l'interlocuteur dans la situation d'énonciation. Un syntagme a été codé comme correspondant à une information ancienne quand cette dernière est retrouvable par l'interlocuteur dans le discours ou dans le contexte. L'information ancienne a par la suite été codée selon sa forme syntaxique : une forme pronominale (6) ou une forme lexicale (7).

(6) Les affaires de ma copine avaient disparues et personne ne savait où elles étaient passées. (3ème $n^{\circ} 05$, narratif écrit, E/O)

(7) Mon frère $m$ 'a conseillé d'acheter le portable d'une fille. Mon frère est venu [...] (3ème $n^{\circ} 01$, narratif écrit, $O / E$ )

Parmi les syntagmes nominaux lexicaux maintenant une information ancienne ont été distingués les SNL maintenant une information de même forme que la mention précédente (répétition) (8) et des SNL maintenant une information ancienne mais impliquant des modifications par rapport à la mention précédente (hyperonymie et synonymie) (9).

(8) Mon père les a injuriés. Une demi-heure plus tard, mon père est parti. (CM2 $n^{\circ} 19$, narratif écrit, $E / O$ )

(9) Un garçon l'embêtait. Donc je suis allé voir ce garçon, l'enfant disait que c'était mon frère. (5ème $n^{\circ} 08$, narratif écrit, $E / O$ ) 


\section{Résultats}

\subsection{Informations liminaires}

Des analyses de variance (ANOVA) ont été effectuées avec : (a) deux facteurs interindividuels, le NIVEAU SCOLAIRE (CM2, $5^{\text {ème }}$ et $3^{\text {ème }}$ ) et l'ORDRE DE PRODUCTION (oral/écrit et écrit/oral) ; et (b) deux facteurs intra-individuels, le TYPE DE TEXTE (expositif et narratif) et la MODALITÉ (orale et écrite). Concernant l'ORDRE DE PRODUCTION, il a été indiqué dans la partie Méthodologie, que notre protocole avait prévu quatre ordres de passation : A, B, C et D. Lors d'études pilotes, nous nous sommes aperçues que le fait de produire un type de texte avant l'autre n'engendre pas de différences significatives, alors que la modalité, oui. De ce fait, les ordres A et C ainsi que les ordres B et D ont été cumulés. Nous avons alors deux types de groupes : les individus ayant débuté par une production écrite (ordre écrit/oral) et ceux ayant débuté par une production orale (ordre oral/écrit). Dans le but de compléter ces analyses de variance, des tests post-hoc PLSD de Fisher ont été effectués, notamment pour le NIVEAU SCOLAIRE. De plus, les interactions entre les facteurs n'étant pas significatives, elles ne sont pas présentées.

\subsection{Le maintien de l'information : pronom ou forme lexicale}

Les proportions des SN maintenant une information sous forme pronominale et lexicale

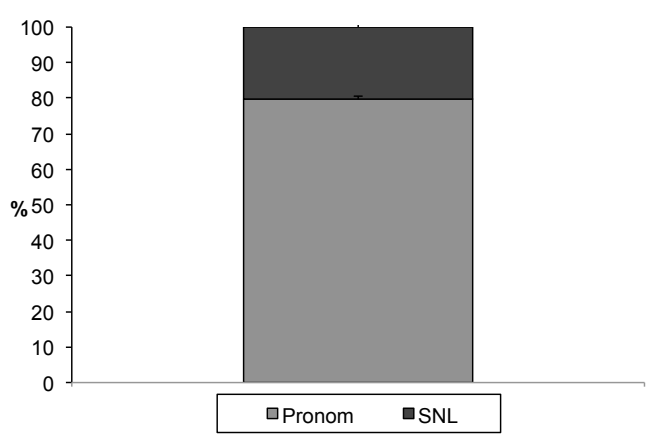

(Fig. 1) sont calculées par rapport au nombre total de SN maintenant une information.

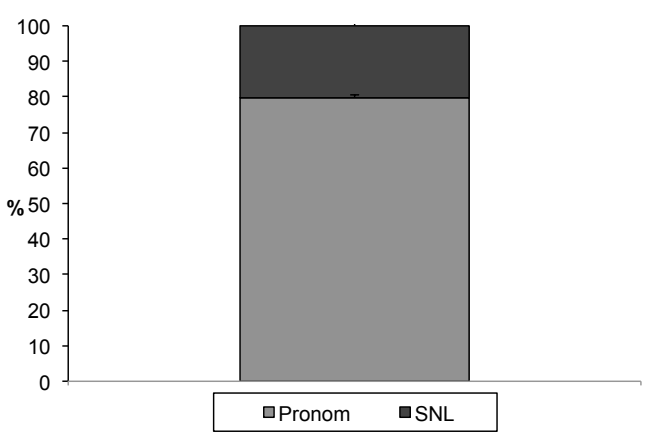

Fig. 1. Proportions des SN ancienne information sous forme pronominale et lexicale

Un référent, une fois introduit, est préférentiellement maintenu par le biais d'une forme pronominale $(79,74 \%)$ que par une forme lexicale $(20,26 \%)$ (Test $\mathrm{T}: \mathrm{t}(503)=29,31, \mathrm{p}<$ $0,0001)$.

De plus, les analyses révèlent que les différences selon le NIVEAU SCOLAIRE $\left(F_{(2,480)}=3,185, \mathrm{p}<0,0423\right)$ sont significatives (Fig. 2). 


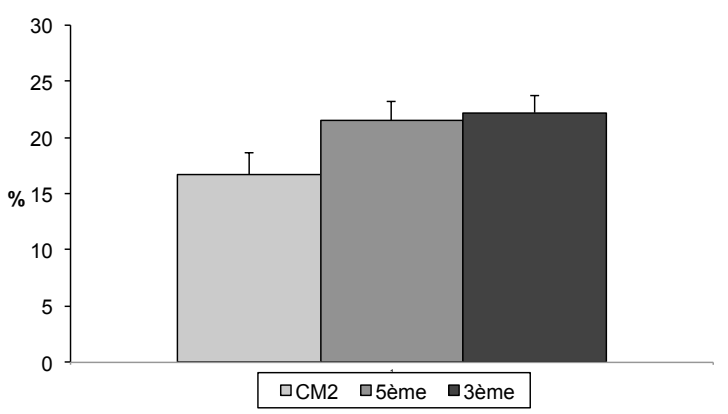

Fig. 2. Proportions des SNL maintenant une information selon le niveau scolaire (par rapport au nombre total de SN maintenant une information)

Les proportions des SNL maintenant une information augmente avec le niveau scolaire. Les écoliers de CM2 ont une proportion de SN maintenant une information sous forme lexicale plus faible $(16,67 \%)$ que les individus de $5^{\text {ème }}(21,53 \%)(\mathrm{p}=0,0427)$ et de $3^{\text {ème }}$ $(22,26 \%)(\mathrm{p}<0,0190)$; les proportions des $5^{\text {ème }}$ et des $3^{\text {ème }}$ ne se distinguant pas significativement $(\mathrm{p}=0,7677)$.

De plus, les différences selon le MODALité $\left(F_{(1,480)}=37,023, \mathrm{p}<0,0001\right)$ sont significatives (Figure 3).

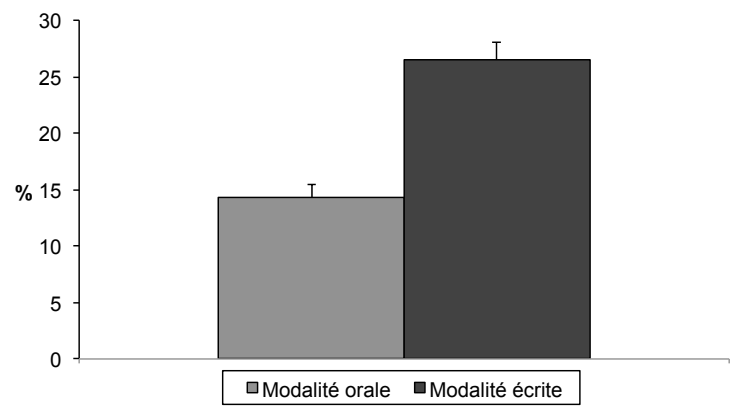

Fig. 3. Proportions des SNL maintenant une information selon la modalité (par rapport au nombre total de SN maintenant une information)

La proportion de SNL pour maintenir un référent constitue davantage une caractéristique de la modalité écrite $(26,48 \%)$ que de la modalité orale $(14,28 \%)$.

Enfin, les différences selon l'ORDRE $\left(F_{(1,480)}=3,563, \mathrm{p}<0,05\right)$ sont significatives (Fig. 4).

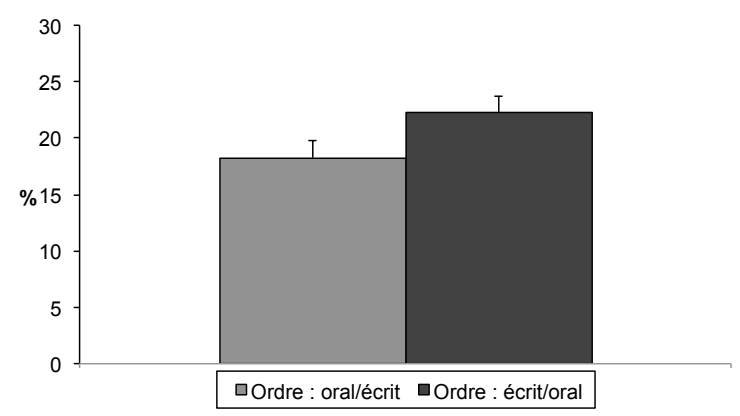

Fig. 4. Proportions des SNL maintenant une information selon l'ordre de production (par rapport au nombre total de SN maintenant une information) 
La proportion de SNL pour maintenir un référent est plus importante dans les textes des individus qui produisent dans l'ordre écrit/oral $(18,27 \%)$ que dans les textes des individus qui produisent dans l'ordre oral/écrit $(22,27 \%)$.

\subsection{Le maintien de l'information sous forme lexicale : quelle forme?}

La Figure 5 présente les proportions des SNL maintenant une information ancienne de même forme que la mention précédente et des SNL maintenant une information ancienne mais impliquant des modifications par rapport à la mention précédente selon le nombre total des SNL maintenant une information, selon les trois groupes d'âge.

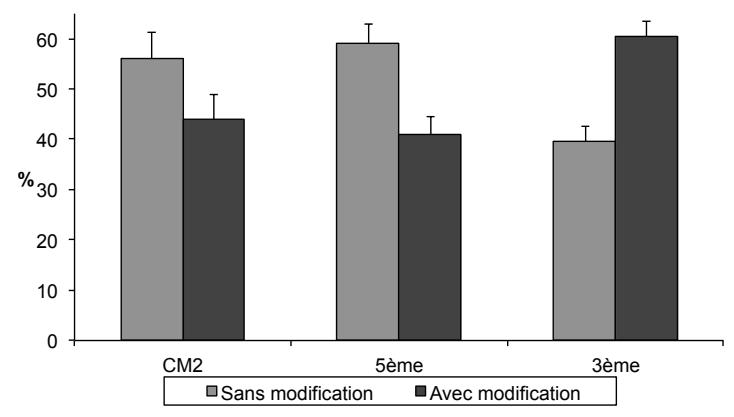

Fig. 5. Proportions des SNL maintenant une information impliquant une répétition ou des modifications linguistiques selon le NIVEAU SCOLAIRE

$\mathrm{Si}$ les écoliers de CM2 et les individus de $5^{\text {ème }}$ produisent davantage de SNL maintenant une information ancienne sans modification (respectivement $56,7 \%$ et $59,9 \%$ ) que de SNL maintenant une information ancienne avec modifications (respectivement $43,3 \%$ et $40,1 \%$ ), les collégiens de $3^{\text {ème }}$, eux, maintiennent davantage une information sous forme lexicale avec modifications $(60,8 \%)$ que sans modification $(39,2 \%)$.

Les analyses révèlent que les différences selon le NIVEAU SCOLAIRE $\left(F_{(2,308)}=9,35, \mathrm{p}<\right.$ 0,0001) sont significatives (Figure 6).

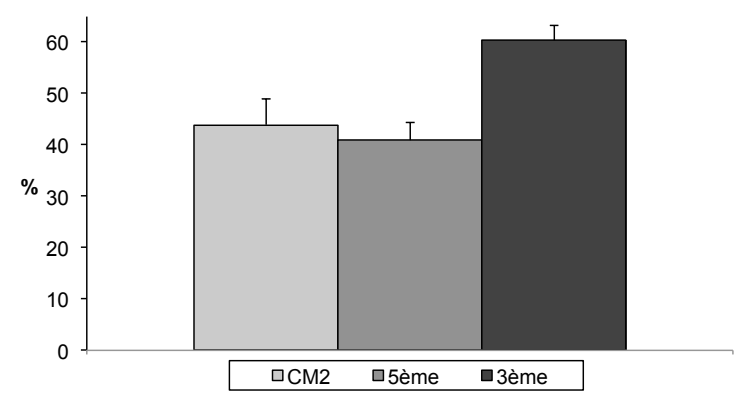

Fig. 6. Proportions des SNL maintenant une information impliquant des modifications linguistiques selon le niveau scolaire (par rapport au nombre total de SNL maintenant une information)

La proportion des SNL maintenant une information ancienne avec modifications augmente avec les années de scolarisation. Les individus de $3^{\text {ème }}$, lorsqu'ils maintiennent une information sous forme lexicale, le font davantage en effectuant des modifications linguistiques qu'en faisant des répétitions; ils maintiennent significativement plus en faisant des modifications $(60,8 \%)$ que les individus de $5^{\text {ème }}(40,1)(p<0,0001)$ et de CM2 $(43,3 \%)(p=0,0031)$ dont les deux proportions ne se distinguent pas significativement $(\mathrm{p}=0,5870)$. 
De plus, les différences en fonction de la MODALITÉ $\left(F_{(1,308)}=10,33, \mathrm{p}<0,0013\right)$ sont significatives (Figure 7).

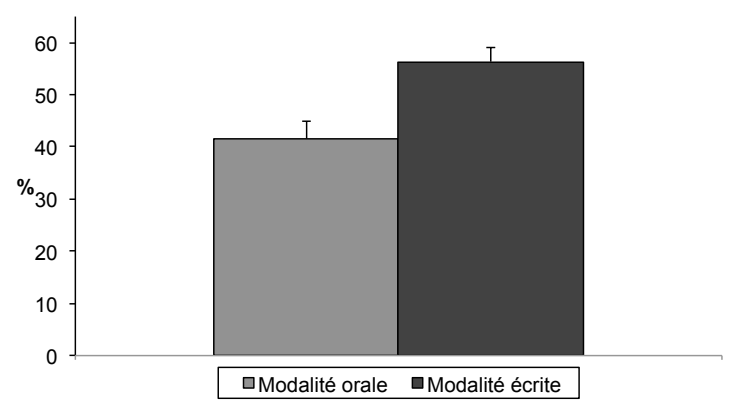

Fig. 7. Proportions des SNL maintenant une information impliquant des modifications linguistiques selon la modalité (par rapport au nombre total de SNL maintenant une information)

La proportion des SNL maintenant une information ancienne avec modifications est plus importante dans les productions écrites $(56,4 \%)$ que les productions orales $(41,6 \%)$.

\section{Discussion}

\subsection{Informations préliminaires}

L'objectif de cet article était d'observer le maintien de l'information ancienne sous forme lexicale dans une perspective développementale. Nous nous demandions à partir de quel âge et dans quel contexte les enfants utilisent une nouvelle forme, le $S N L$, pour une ancienne fonction, maintenir une information? Pour ce faire, deux variables principales ont été observées : la proportion des SNL maintenant une information (Figures 1 à 4) et la proportion du maintien sous forme lexicale impliquant des changements linguistiques selon les facteurs - NIVEAU SCOLAIRE, TYPE DE TEXTE, MODALITÉ et ORDRE -.

Le premier résultat révèle que la forme la plus sollicitée pour encoder un référent déjà introduit dans un texte est le pronom (Figure 1). Ce résultat est trivial dans la mesure où il confirme de nombreuses théories et données allant dans ce sens (Ariel, 1996 ; Chafe, 1994 ; Fox, 1987 ; Khorounjaia et al., 2004 ; Mazur-Palandre, 2015 ; Mazur-Palandre et Jisa, 2012). Les pronoms sont utilisés préférentiellement pour encoder un référent de haute accessibilité ; en revanche, les SNL encodent préférentiellement des référents de basse accessibilité. L'Hypothèse 1 est donc confirmée.

\subsection{Le maintien de l'information sous forme lexicale : une acquisition tardive}

Dans les productions observées, 20,26\% des référents sont maintenus par le biais d'une forme lexicale (Figure 1). Une de nos prédictions était un effet significatif du NIVEAU SCOLAIRE : avec les années de scolarisation, les individus de notre étude maintiendraient de plus en plus une information sous forme lexicale. Ceci est confirmé par nos résultats (Figure 2), qui valide 1'Hypothèse 2. Les individus développent leur répertoire, ils utilisent alors une nouvelle forme, le $S N L$, pour une ancienne fonction, maintenir une information. Rappelons que les écoliers de CM2 (10) maintiennent significativement moins l'information ancienne sous forme lexicale que les deux populations de collège (11) ; ces deux derniers groupes ne se distinguent guère.

(10) C'était avec un copain, j'avais ramené un cahier, il a voulu le prendre. (CM2 $n^{\circ} 42$, narratif écrit, $E / O$ ) 
Elle n'avait pas vu qu'elle avait perdu ses sous. Moi, j'ai ramassé l'argent et je lui ai rendu ses sous. (5ème $n^{\circ} 47$, narratif écrit, E/O)

Dans ces exemples, l'écolier introduit les informations nouvelles un copain et un cahier, puis les maintient par les pronoms $i l$ et le. Le collégien introduit les référents ses sous puis le maintient deux fois sous forme lexicale, l'argent et ses sous.

Le choix naturel et le plus économique, qui est lié aux principes de transmission de l'information de l'oral, est l'encodage sous forme pronominale (Ariel, 1996; Fox, 1987 ; Khorounjaia et Tolchinsky, 2004). Avec l'expérience textuelle et le perfectionnement de l'écrit, les enfants répondent de moins en moins à ce précepte. Produire des SNL exige un coût cognitif important et notamment lorsqu'il faut les mettre en position sujet (MazurPalandre et Jisa, 2013 ; Mazur-Palandre, 2015). Ainsi, produire un SNL pour maintenir une information exige des individus (a) non seulement un effort pour l'accès lexical mais aussi (b) d'aller à l'encontre d'un principe bien connu et intégré : l'encodage de l'information ancienne sous forme pronominale.

La proportion moyenne des SNL maintenant une information ancienne sous forme lexicale ne semble pas évoluer entre les différents âges. Cependant, la Figure 5 montre que, si nous regardons la structure de ces SNL, alors une différence de proportion entre les trois groupes d'âge est observable: les enfants de CM2 et $5^{\text {ème }}$, quand ils maintiennent une information sous forme lexicale le font davantage sans changement de forme entre la première mention et la seconde (donc ils ont une préférence pour la répétition) qu'avec des changements linguistiques (synonymie et hyperonymie). En revanche, la tendance s'inverse pour les adolescents $\left(3^{\text {ème }}\right)$. Les analyses montrent, de plus, que la différence de proportion de maintien sous forme lexicale impliquant des changements linguistiques (synonymie et hyperonymie) entre les trois populations est significative : ce type de maintien augmente de façon générale avec le NIVEAU SCOLAIRE (Figure 6) (12). Ce résultat confirme alors l'Hypothèse 2bis: les enfants maintiennent de plus en plus l'information sous forme lexicale par le biais de la synonymie ou l'hyperonymie et moins par le biais de la répétition. (12) Et après j(e) l'ai dit à ma mère / ma mère elle est venue. (CM2 $n^{\circ} 08$, narratif oral, $E / O$ )

L'enfant introduit le nouveau référent ma mère et le maintient dans la proposition suivante sous forme lexicale sans faire de modification linguistique. La différence de proportion entre les $\mathrm{CM} 2$ et les $5^{\text {ème }}$ ne se révèle pas significative. Néanmoins, il apparaît que les $3^{\text {ème }}$ en produisent significativement plus que les deux plus jeunes populations : les collégiens de $3^{\text {eme }}$ maintiennent plus une information en variant la forme linguistique alors que les deux autres populations se contentent de répéter le lexème en question (13).

(13) On faisait un exercice individuellement quand ma copine m'a demandé si j'avais utilisé la première méthode pour répondre au problème. $\left(3^{\text {ème }} n^{\circ} 39\right.$, narratif écrit, $O / E)$

La collégienne introduit une information nouvelle, un exercice, puis la maintient sous une forme lexicale impliquant des changements linguistiques, au problème.

De plus, une étude qualitative des SNL maintenant une information en impliquant des changements linguistiques révèle que les modifications des CM2 sont minimes par rapport à celles effectuées par des $3^{\text {ème }}$. En effet, les individus les plus jeunes réalisent des modifications sur les déterminants (14). En revanche, les participants les plus âgés font des modifications exigeant plus d'efforts cognitifs telle que la substitution lexicale (15). Les collégiens de $3^{\text {ème }}$ font preuve de flexibilité et d'ouverture de leur répertoire linguistique alors que les CM2 sont restreints à des changements morphologiques.

(14) Une fille s'était mise avec moi dans une chambre avec d'autres filles aussi cette fille s'énervait contre sans arrêt pour rien. (CM2 $n^{\circ} 08$, narratif écrit, E/O)

(15) Ça s'appelle une antisèche, ça prouve qu'on n'a pas appris nos leçons. Si le ou la professeur voit le papier dans la trousse, il va peut être mettre une heure de colle ou une sanction plus grave. ( $3^{\text {ème }} n^{\circ} 44$, expositif écrit, E/O) 
Une seconde remarque qualitative concernant le rôle de ces maintiens lexicaux peut être ajoutée. Le maintien lexical, que ce soit avec ou sans modification, peut avoir divers rôles. Si les plus jeunes maintiennent sous forme lexicale en créant de la redondance (16), les plus âgés se servent du maintien lexical pour créer des effets de style ou encore pour désambigüiser des situations (17).

(16) Ma mère est arrivée et il a menti à ma mère et ma mère l'a cru. (CM2 $n^{\circ} 08$, narratif écrit, $E / O$ )

(17) Il y a quelques jours, j'ai acheté un nouveau téléphone portable sur internet. Ce $n$ 'est pas du tout pratique car il y a trop de problèmes. En fait, j'en ai rencontrés plusieurs : lors de la livraison du colis, il n'eu pas l'objet que j'avais commandé. [...] Il y eut également un autre problème avec le téléphone. $\left(3^{\text {ème }} n^{\circ} 24\right.$, narratif écrit, $O / E)$

Le CM2 utilise la répétition pour maintenir le référent ma mère crée de la redondance. En revanche, le maintien de l'information sous forme lexicale réalisé par un individu de $3^{\text {ème }}$ n'a pas cet effet (17). Dans cet exemple, le référent un nouveau téléphone portable est maintenu par une substitution lexicale, l'objet. Puis, de nouveau l'individu apporte des modifications linguistiques lors de la mention suivante, le téléphone, et ce maintien lexical a pour but de réintroduire cet élément qui aurait pu être désactivé ; il prend en considération que la distance entre les deux mentions peut impliquer un problème de compréhension si le référent est maintenu avec un pronom.

\subsection{Le maintien de l'information sous forme lexicale : un choix linguistique typique de l'écrit formel}

Une autre de nos attentes était un effet significatif du contexte de production (Hypothèses 3 et 3 bis) ce qui est confirmé, en partie, par nos résultats (Figures 3 et 7). En effet, seule la MODALITÉ a un effet significative sur les variables observées. Maintenir un référent par le biais d'un SNL correspond davantage aux textes produits à l'écrit (19), d'autant plus si ce maintien implique un changement de forme lexicale par rapport à la mention précédente (à savoir l'utilisation de la synonymie et de l'hyperonymie). Le fait de maintenir une information sous forme lexicale exige d'aller à l'encontre des principes naturels de la transmission de l'information, et notamment ceux de l'oral. Ceci implique alors un effort cognitif important qui est facilité en production écrite par son temps de planification important. De plus, la production de SNL est beaucoup plus aisée à l'écrit qu'à l'oral ; en effet, la production de SNL est coûteuse et les individus trouvent davantage de ressources à l'écrit. Les exemples (18) et (19) ci-dessous sont représentatifs de cette situation.

(18) J'avais changé un peu radicalement de style vestimentaire. On s'est revu voilà il a vu que mentalement j'avais pas changé. ( $3^{\text {ème }} n^{\circ} 27$, narratif oral, O/E)

(19) Il y a quelques années, il m'est arrivé une dispute avec mon oncle à cause de mon changement vestimentaire. [...] Il avait fait une erreur en s'arrêtant sur mon nouveau style vestimentaire. ( $3^{\text {eme }} n^{\circ} 27$, narratif écrit, $O / E$ )

Le collégien introduit à l'écrit une information nouvelle mon changement vestimentaire qu'il maintient par le biais du SNL mon nouveau style vestimentaire. Ce même individu introduit à l'oral également un nouveau SNL, style vestimentaire, mais ne le maintient pas.

De plus, les individus maintiendraient plus fréquemment une information en variant la forme linguistique dans les textes écrits, modalité écrite qui, notamment par son temps de planification important, donne la possibilité aux locuteurs/scripteurs d'utiliser un type d'encodage se réalisant moins aisément en production orale. Les locuteurs/scripteurs apprennent à aller à l'encontre de certains principes de transmission de l'information : ils produisent des SNL dans le but de maintenir l'information. 


\subsection{Les enfants parlent comme ils écrivent}

L'ORDRE DE PRODUCTION affecte également la proportion de l'information ancienne sous forme lexicale ainsi que le maintien sous forme lexicale impliquant des changements linguistiques, ce qui va dans le sens de nos Hypothèses (4 et 4bis). Le maintien de l'information par le biais d'un SNL est plus fréquent dans les textes d'individus ayant produit dans l'ordre écrit/oral (20) et (21).

(20) Alors un jour, elle a lu mes $\boldsymbol{S}_{-} \boldsymbol{M}$ S S personnels. Alors pendant une période j'avais peur que mes parents prennent mon téléphone pour lire mes messages. ( $3^{\text {ème }} n^{\circ} 14$, narratif écrit, E/O)

(21) Ben entre temps elle a vu mes messages et tout et donc elle a lu des messages euh personnels. ( $3^{\text {ème }} n^{\circ} 14$, narratif oral, E/O)

L'utilisation de formes lexicales pour maintenir un référent (mes SMS personnels $\sim$ mes messages), caractéristique de l'écrit, se trouve transférée à l'oral (mes messages $\sim$ des messages).

Le fait de produire un texte écrit puis un texte oral favorise le maintien sous forme lexicale étant donné que l'écrit facilite la proportion de SNL ; à l'écrit, les individus ont le temps d'aller au-delà des principes de transmission de l'information de l'oral. Une fois cette stratégie (maintenir un SNL par un autre SNL) activée et appliquée à l'écrit, elle est transférée à l'oral. Néanmoins, si le sujet commence par faire un texte oral, il tend à respecter les tendances naturelles du traitement de l'information en maintenant l'information ancienne sous forme pronominale dans la mesure où la modalité orale ne permet pas de les transgresser. Cette attitude se répercute en production écrite.

\section{Conclusion}

Les participants de notre étude obéissent au principe de transmission de l'information selon lequel le maintien de l'information se réalise préférentiellement sous forme pronominale (Ariel, 1996 ; Allen et al., 2008 ; Fox, 1987 ; Khorounjaia et Tolchinsky, 2004 ; MazurPalandre et Jisa, 2012; Mazur-Palandre, 2015). Les analyses révèlent également que le maintien sous forme lexicale varie selon trois facteurs de ce travail de recherche - NIVEAU SCOLAIRE, MODALITÉ et ORDRE DE PRODUCTION-. La proportion des SNL maintenant une information augmente avec l'âge, et est plus importante dans les textes écrits et dans les productions réalisées par des participants appartenant à l'ordre écrit/oral. Le maintien sous forme lexicale est non naturel dans le sens où il exige d'aller à l'encontre des principes de transmission de l'information de l'oral. Ce type de maintien, qui apparaît comme un indice de maturité linguistique, se réalise de plus en plus fréquemment, en impliquant notamment des formes diverses. La substitution lexicale dépend de la capacité de l'enfant à produire un SNL, ce qui représente un effort cognitif plus important que l'utilisation d'un pronom, pour un référent déjà connu. Ce choix linguistique exige également de l'enfant de comprendre que, selon le contexte de production, il peut être utile, pour créer un effet de style, d'aller à l'encontre d'un des principes de transmission de l'information : maintenir une information avec un pronom. Les adolescents de $3^{\text {ème }}$, avec l'expérience de l'écrit et de différents types de textes, arrivent à aller à l'encontre de ce principe de transmission de l'information de l'oral. Le cheminement vers la capacité de maintenir une information par le biais lexical est ainsi bien engagé.

\section{Références bibliographiques}

Aksu-Koç, A. et Kuntay, A. (2001). Reformulating causal relations while retelling narratives: evidence from Turkish. Colloque Acquisition et construction du sens dans une perspective interlangue, Paris, décembre 2001. 
Allen, S., Skarabela, B. et Hughes, M. (2008). Using corpora to examine discourse effects in syntaxe. Trends in language acquisition research, Corpora in language acquisition research, 6.

Ariel, M. (1996). Referring expressions and the $+/$ - coreference distinction, In Fretheim, T. et Gundel, J. (eds.), Reference and referent accessibility, pp. 13-37. Amsterdam/Philadelphia : John Benjamins.

Bellert, I. (1971). On a condition of the coherence of texts, Semiotica, 4, 253-288.

Berman, R. (2008). The psycholinguistics of text construction. Journal of Child Language, $35,1-37$.

Berman, R. et Nir-Sagiv, B. (2007). Comparing narrative and expository text construction across adolescence: A developmental paradox. Discourse Processes, 43(2), 79-120

Berman, R. et Ravid, D. (2009). Becoming a literate language user oral and written text construction across adolescence, In Olson, D., Torrance, N. (eds.), The Cambridge Handbook of Literacy, 92-111, Cambridge University Press.

Berman, R. et Slobin, D. (1994). Relating events in narrative: a crosslinguistic developmental study. Hillsdale, Erlbaum.

Berman, R. et Verhoeven, L. (2002). Cross linguistic perspectives on the development of text-production abilities in speech and writing, Written Language and Literacy, 5, Vol.1.

Blanche-Benveniste, C. (1983). L'importance du français parlé pour la description du français tout court. Recherches sur le Français Parlé, 5, 23-45.

Blanche-Benveniste, C. (1990). Le français parlé. Paris: Edition du Centre National de la Recherche Scientifique.

Blanche-Benveniste, C. (2000). Approche de la langue parlée en français. Gap: Ophrys.

Boscolo, P. (1990). The construction of expository text. First Language, 10, 217-230

Britton, B. (1994). Understanding expository text: Building mental structures to induce insights. In Gernsbacher, M. (ed.), Handbook of Psycholinguistics, pp. 641-674, San Diego, California, Academic Press.

Bruner, J. (1986). Actual Minds, possible worlds. Cambridge, Harvard University Press.

Chafe, W. (1994). Discourse, consciousness, and time: The flow and displacement of conscious experience in speaking and writing. Chicago, University of Chicago Press.

Charolles, M. (1978). Introduction aux problèmes de la cohérence des textes. Langue Française, 38, 7-41.

Clark, H. et Haviland, S. (1977). Comprehension and the Given-New contract. In Freedle, R. (ed.), Discourse Production and Comprehension, pp. 1-40. Norwood, NJ: Ablex.

De Weck, G. (1991). La cohésion dans les textes d'enfants, étude du développement des processus anaphoriques. Neuchâtel: Delachaux et Niestlé.

De Weck, G. et Schneuwly, B. (1994). Anaphoric procedures in four text types written by children. Discourse Processes, 17, 101-113.

Du Bois, J. (1987). The discourse basis of ergativity. Language, 63, 805-855. 
Du Bois, J. (2003). Argument Structure: Grammar in use. In Du Bois, J., Kumpf, L., Ashby, W. (eds.), Prefered Argument Structure: Grammar as architecture for function, pp. 11-60, Amsterdam: John Benjamins.

Fayol, M. (1997). Des idées au texte: psychologie cognitive de la production verbale orale et écrite. Paris, Presse Universitaire de France.

Fox, B. (1987). Discourse structure and anaphora.Cambridge: Cambridge University Press.

Gadet, F. (1996). Une distinction bien fragile : oral/écrit. TRANEL (Travaux neuchâtelois de linguistique), 25, 13-27.

Gayraud, F. (2000). Le développement de la différenciation oral/écrit vu à travers le lexique. Doctorat, Sciences du Langage, Université Lumière

Gayraud, F., Jisa, H. et Viguié, A. (2001). Utilisation des outils cohésifs comme indice de sensibilité au registre : une étude développementale. AILE, 14, 3-24.

Halliday, M. et Hasan, R. (1976/1989). Cohesion in English. Londres: Longman group limited.

Hickmann, M. (2002). Développement de la production verbale orale. In Fayol, M. (ed.), Production du langage, pp.173-203. Paris: Lavoisier.

Hickmann, M. (2003). Children's discourse: person, space and time across languages. Cambridge ; New York, Cambridge University Press.

Hickmann, M. et Hendriks, H. (1999). Cohesion and anaphora in children's narratives : a comparison of English, French, German and Mandarin Chinese. Journal of Child Language, 26, 419-452.

Hickmann, M., Hendriks, H., Roland, F. et Liang, J. (1996). The marking of new information in children's narratives: a comparison of English, French, German, and Mandarin Chinese. Journal of child Languages, 23, 591-619.

Jisa, H. (2000). Increasing cohesion in narratives: a developmental study of maintaining and reintroducing subjects in French, Linguistics, 38, 591-620.

Jisa, H. (2004). A developmental study of discourse stance in spoken and written French. Writing 2004, Genève, 20-22 septembre.

Jisa, H. et Mazur, A. (2006), "L'expression de la causalité : une étude développementale". Acte Journée d'étude : Des savoirs savants aux savoirs enseignés, Université Paris X, Nanterre, Presses universitaires de Namur, 33-60.

Kail, M. et Hickmann, M. (1992). French children's hability to introduce referents in narratives as a function of mutual knowledge. First Language, 12, 637-62.

Katzenberger, I. (2004). The development of clause packaging in spoken and written texts. Journal of Pragmatics, 36, 1921-1948.

Katzenberger, I. (2005). The super-structure of written ewpository texts - a developmental perspective. In Ravid, D. et Shyldkrot, H.B. (eds.), Perspectives on language and language development. Essays in Honor of Ruth A. Berman, pp. 327-336. Dordrecht, Kluwer Academic Publishers.

Khorounjaia, E. et Tolchinsky, L. (2004). Discursive constraints on the lexical realization of arguments in Spanish. In Berman, R. (ed.), Language Development across Childhood and Adolescence, Vol. 3, pp. 83-110. Amsterdam/Philadelphia: John Benjamins. 
Lambrecht, K. (1994). Information structure and sentence form: topic, focus and mental representations of discourse referents, Cambridge: Cambridge University Press.

Levelt, W. (1989). Speaking: From intention to articulation. Cambridge/Massachussetts: The MIT Press.

Mazur-Palandre, A. (2010). Formes et positions syntaxiques du syntagme nominal : Une étude développementale du français scolaire en ZEP. Sciences Croisées, Numéro thématique.

Mazur-Palandre, A. (2015). Overcoming Preferred Argument Structure in written French: development, modality, text type. Written Language and Literacy, 18:1, 25-55.

Mazur-Palandre, A. et Jisa, H. (2013). La lexicalité des productions orales et écrites : Une étude développementale. Enfance, 4, 359-371.

Mazur-Palandre, A. et Jisa, H. (2012). Introduire et développer l'information : une acquisition tardive? CogniTextes 7.

Moirand, S. (1975). Le rôle anaphorique de la nominalisation dans la presse écrite. Langue Française, 28, 60-78.

Mosenthal, P. (1985). Défining the expository discourse continuum, toward a taxinomy of expository text. Poetics, 14, 387-414.

Pellegrini, A., Galda, L. et Rubin, D. (1984). Context in text: The development of oral and written language in two genres. Child Development, 55, 1549-1555.

Ravid, D. (2005). Emergence of linguistic complexity in later language development:evidence from expository text construction. In Ravid, D., Shyldkrot, H.B. (eds.), Perspectives on language and language development. Essays in Honor of Ruth A. Berman, pp. 337-355. Dordrecht, Kluwer Academic Publishers.

Ravid, D. et Berman, R. (2010). Developing noun phrase complexity across adolescence: A text-embedded analysis,. First Language, 30(1), 1-29.

Reinhart, T. (1980). Conditions for text coherence. Poetics Today, 1, 161-180.

Sanford, A. et Garrod, S. (1981). Understanding written language. New York: Wiley.

Schneuwly, B. (1988). Le langage écrit chez l'enfant. Neuchâtel: Delachaux et Niestlé.

Schneuwly, B., Rosat, M.C. et Dolz, J. (1989). Les organisateurs textuels dans 4 types de textes écrits. Étude chez des élèves de 10, 12 et 14 ans. Langue Française, 81, 40-58.

Tolchinsky, L. (2006). The emergence of writing, In MacArthur, C., Graham, S., Fitzgerald, J. (eds.), Handbook of Writing Research, 83-95, New York: The Guilford Press.

Tolchinsky, L., Perera, J., Argerich, N. et Aparici, M., (1999). Rhetorical moves and noun phrase complexity in written expository texts. Developing Literacy Across Genres, Modalities and Languages, 1, 257-278.

Viguié-Simon, A. (2001). Développement de la connectivité selon le type textuel et le mode de production au cours de l'adolescence, Thèse de Doctorat, Université Lumière, Lyon. 
${ }^{1}$ Les auteurs remercient le LabEx Aslan (ANR-10-LABX-0081) de l'Université de Lyon pour son soutien financier dans le cadre du programme « Investissements d'Avenir » (ANR-11-IDEX-0007) de l’État Français géré par l'Agence Nationale de la Recherche (ANR).

${ }^{2}$ Le terme « proposition » est utilisé pour faire référence à la notion de clause telle qu'elle est définie par Berman et Slobin (1987:7): " any unit that contains a unified predicate. By unified, we mean a predicate that expresses a single situation (activity, event, state). Predicates include finite and nonfinite verbs as well as predicate adjectives. In general, clauses will be comprised of a single verbal element; however, infinitives and participles which function as complements of modals or aspectual verbs are included with the matrix verb as a single clause ». 